\title{
Counting the Number of Glutamate Molecules in Single Synaptic Vesicles
}

Yuanmo Wang, Hoda fathali, devesh mishra, Thomas Olsson, Jacqueline Keighron, karolina skibicka, Ann-Sofie Cans

Submitted date: 30/08/2019 - Posted date: 03/09/2019

Licence: CC BY-NC-ND 4.0

Citation information: Wang, Yuanmo; fathali, Hoda; mishra, devesh; Olsson, Thomas; Keighron, Jacqueline; skibicka, karolina; et al. (2019): Counting the Number of Glutamate Molecules in Single Synaptic Vesicles. ChemRxiv. Preprint.

Analytical tools for direct quantitative measurements of glutamate, the principal excitatory neurotransmitter in brain, are lacking. Here, we introduce a new enzyme-based amperometric sensor technique for direct counting of the number of glutamate molecules stored inside single synaptic vesicles. An ultra-fast enzyme-based glutamate sensor is placed into a solution of isolated synaptic vesicles, which stochastically rupture at the sensor surface in a potential dependent manner by applying a constant negative potential. High-speed $(10 \mathrm{kHz})$ amperometry is used to record sub-millisecond current spikes, which represent glutamate release from single vesicles that burst open. Glutamate quantification is achieved by a calibration curve that is based on measurements of glutamate release from vesicles pre-filled with various concentrations of glutamate. Our measurements show that a single synaptic vesicle encapsulates about 8000 glutamate molecules, which is comparable to the measured exocytotic quantal glutamate release in the nucleus accumbens of mouse brain tissue. Hence, this new methodology introduces the means to quantify ultra-small amounts of glutamate and to study synaptic vesicle physiology, pathogenesis and drug treatments for neuronal disorders where glutamate is involved.

File list (2)

Cans_Manuscript.docx (6.79 MiB)

view on ChemRxiv • download file

Cans_Supporting Information.docx (4.48 MiB)

view on ChemRxiv • download file 


\title{
Counting the Number of Glutamate Molecules in Single Synaptic Vesicles
}

\author{
Yuanmo Wang, ${ }^{\dagger}$ Hoda Fathali, ${ }_{-}^{\dagger}$ Devesh Mishra, ${ }^{\ddagger}{ }^{\perp}$ Thomas Olsson, \\ Keighron ${ }^{\#}$, Karolina P. Skibicka, ${ }^{\perp, \neq}$ and Ann-Sofie Cans*,† \\ ${ }^{\dagger}$ Department of Chemistry and Chemical Engineering, Chalmers University of Technology, \\ Kemigården 4, SE-412 96 Gothenburg, Sweden; \\ ‡Department of Physiology/Metabolic Physiology, Institute of Neuroscience and Physiology, The \\ Sahlgrenska Academy at the University of Gothenburg, Medicinaregatan 11, SE-413 90 Gothenburg, \\ Sweden; \\ "Department of Chemical and Biological Sciences, New York Institute of Technology, Old Westbury, \\ NY 11568, USA \\ "Department of Physics, Chalmers University of Technology, Kemigården 4, SE-412 96 Gothenburg, \\ Sweden; \\ ${ }^{\perp}$ Wallenberg Centre for Molecular and Translational Medicine, University of Gothenburg, SE-405 30 \\ Gothenburg, Sweden
}

Supporting Information Placeholder

ABSTRACT: Analytical tools for direct quantitative measurements of glutamate, the principal excitatory neurotransmitter in brain, are lacking. Here, we introduce a new enzyme-based amperometric sensor technique for direct counting of the number of glutamate molecules stored inside single synaptic vesicles. An ultra-fast enzymebased glutamate sensor is placed into a solution of isolated synaptic vesicles, which stochastically rupture at the sensor surface in a potential dependent manner by applying a constant negative potential. High-speed $(10 \mathrm{kHz})$ amperometry is used to record submillisecond current spikes, which represent glutamate release from single vesicles that burst open. Glutamate quantification is achieved by a calibration curve that is based on measurements of glutamate release from vesicles pre-filled with various concentrations of glutamate. Our measurements show that a single synaptic vesicle encapsulates about 8000 glutamate molecules, which is comparable to the measured exocytotic quantal glutamate release in the nucleus accumbens of mouse brain tissue. Hence, this new methodology introduces the means to quantify ultra-small amounts of glutamate and to study synaptic vesicle physiology, pathogenesis and drug treatments for neuronal disorders where glutamate is involved.

Synaptic vesicles are organelles used by brain cells to tightly pack and store neurotransmitters in the cell cytoplasm. $^{1}$ Neurotransmitter signals are exchanged between neurons through electrical depolarization which trigger these vesicles to fuse with the cell plasma membrane and release the signaling molecules into the synaptic cleft in a process called exocytosis. From here the neurotransmitters diffuse and bind to receptors at the cell surface of neighboring neurons to relay the chemical message. The amount of neurotransmitter released and the frequency of exocytosis activity are how synapses are thought to control communication strength through plasticity. This process underlies the basics of brain functions such as memory and learning, and also relates to brain disorders including drug abuse and addiction, diseases still not fully understood. ${ }^{2}$ Therefore, many drugs are designed to affect the quantity of neurotransmitters released and residing in the extracellular space to enhance the chemical signaling process. 
Glutamate is the major excitatory neurotransmitter involved in major brain functions such as cognition, memory and learning as well as many neurological disorders. ${ }^{1}$ As such, glutamate regulation through pharmacological agents is of specific interest in managing disease states. Specifically, the amount of neurotransmitters stored in a synaptic vesicle, often referred to as the vesicle "quantal size", is an important quantitative measure for neuropharmacology and drug development research. ${ }^{3-7}$ However, there is still an analytical challenge for direct, reliable quantitative measurements of glutamate stored in the ultra-small volume of a synaptic vesicle with an average diameter of $40 \mathrm{~nm}^{8,9}$ Methods such as radioreceptor assay (3640 glutamate molecules/vesicle) ${ }^{10}$ and immunoisolation of synaptic vesicles from synaptosomes $(60 \mathrm{mM})^{11}$ have been used to try to solve the debate about how many glutamate molecules are stored inside synaptic vesicles. While methods such as fluorescence imaging techniques are powerful tools for studying the mechanisms for vesicular glutamate loading and storage. ${ }^{9,12,13}$ To gain a better understanding of neurophysiology, neurological diseases and possible treatments, methods for direct quantification of synaptic vesicle glutamate storage and its release is needed. Recently, new methodologies have been developed for the quantitative analysis of catecholamine containing vesicles using amperometry. ${ }^{14,15}$ These techniques place a carbon fiber microelectrode into a solution of isolated secretory vesicles and apply a positive oxidation potential that triggers vesicles in contact with the electrode surface to stochastically rupture. Catecholamines that leak out from vesicles that bursts open and onto the electrode surface are electrochemically oxidized and these events are recorded as isolated current spikes by the amperometric electrode. The integrated total charge (Q) for each current spike is used to calculate the vesicle neurotransmitter concentration, through Faraday's law. ${ }^{14,16}$ The mechanism for this method is influenced by a combination of vesicle adsorption and a positive applied potential, which initiates vesicle membrane pore formation that finally shatters the organelle. ${ }^{17}$ Granting amperometry is a quantitative method that displays excellent temporal resolution, the limitation is that it only applies for neurotransmitters that are electroactive.

Glutamate is a non-electroactive neurotransmitter that cannot directly be detected amperometrically, but this becomes feasible by functionalizing the electrode surface with an enzyme, glutamate oxidase (GluOx), creating a glutamate biosensor. ${ }^{18}$ Here, the enzyme works to catalyzes glutamate into forming hydrogen peroxide, $\mathrm{H}_{2} \mathrm{O}_{2}$, which serves as an electroactive redox reporter molecule for glutamate detection. Glutamate sensing has long suffered from low temporal resolution and this prevented applications such as monitoring neuronal single exocytosis activity in real time. To solve this, an ultrafast glutamate sensor, made of a gold nanoparticle (AuNP) modified carbon fiber microelectrode with an ultrathin coating of GluOx, was recently developed in our lab. ${ }^{19}$ This invention helped to push the temporal limits for a chemically selective glutamate sensor from previous sub-second to current sub-millisecond time scale. ${ }^{19}$ The sensor detection scheme (Figure $1 A$ ) is based on the electrochemical reduction reaction for $\mathrm{H}_{2} \mathrm{O}_{2}$ detection by applying an amperometric reduction potential at $-0.5 \mathrm{~V}$ ( $\mathrm{vs}$ a $\mathrm{Ag} / \mathrm{AgCl}$ reference electrode) at the sensor surface. By utilizing this technical achievement, the dynamics of individual sub-millisecond exocytosis events during spontaneous glutamate activity at the core region of the nucleus accumbens in rodent brain tissue was temporally resolved. ${ }^{19}$ Although relative quantitative measures were feasible, the sensor was not validated for absolute quantification. In order to address the lack of methods for quantifying ultra-small amounts of glutamate, we first developed a new method for quantification of glutamate in small synaptic vesicles. This was achieved by implementing our ultrafast enzyme-based glutamate sensor and adopting to previous protocols for quantitative analysis of vesicles containing easily oxidized neurotransmitters. ${ }^{14,15,17}$ Briefly, we placed our sensor into a solution of either glutamate-filled large unilamellar vesicles (LUVs) or isolated synaptic vesicles from mouse brain. In contrast to the catecholamine analysis protocols using positive oxidation potentials at a bare carbon fiber microelectrode, we applied a constant 
negative reduction potential of $-0.5 \mathrm{~V}$ (vs a $\mathrm{Ag} / \mathrm{AgCl}$ reference electrode) at the sensor surface to avoid oxidative interfering analytes and produce chemically selective measurements. This strategy resulted in amperometric time traces that demonstrated stochastic bursts of individual submillisecond reduction current spikes corresponding to detection of single LUVs (Figure 1B) and synaptic vesicles (Figure 1C) randomly bursting and releasing their internal glutamate content onto the sensor surface. In both of these amperometric time traces, the current spikes displayed a rapid current rise and a slower decay versus time (Table S1 and S2), which are typical shape characteristics for detection of vesicle content release (Figure S1A) and indicates that a similar mechanism is acting to rupture these different vesicle compartments. ${ }^{20}$
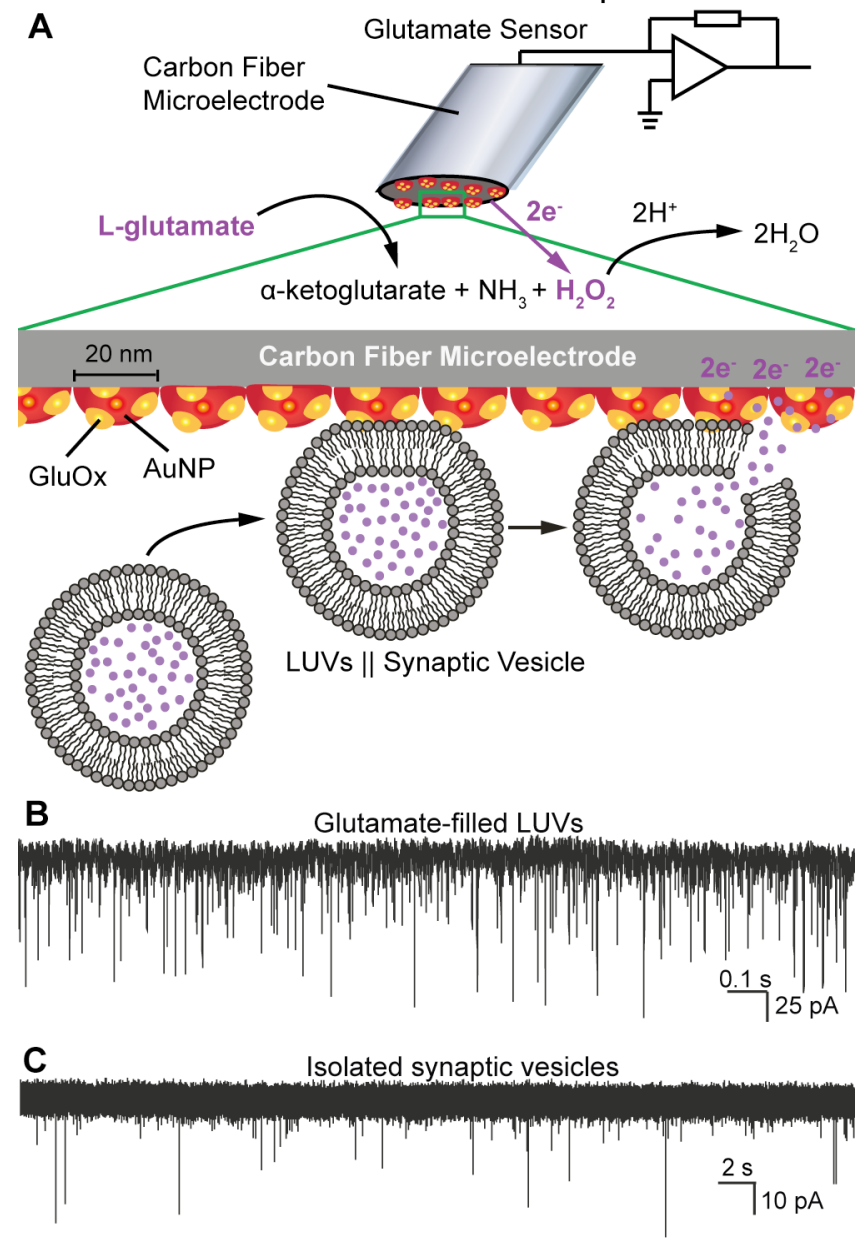

Figure 1. (A) Schematic of the ultrafast glutamate sensor detection scheme used for counting the number of glutamate molecules in single synaptic vesicles as vesicles rupture and release their glutamate content at the sensor surface, modified with AuNPs (red hemispheres) and an ultra-thin coating of GluOx (yellow hemispheres). Schematic is not drawn to scale. (B) Amperometric reduction current recording of glutamate release from LUVs encapsulating 100 mM glutamate, and (C) isolated synaptic vesicles from mouse brain.

Empirical titration of the LUVs and synaptic vesicle samples was performed to find the threshold of vesicular concentration needed to initiate vesicle rupture at the electrode surface. A significantly higher frequency was observed for LUVs compared to synaptic vesicles, which is in agreement with the idea that the protein-rich synaptic vesicle membrane is substantially more rigid and more difficult to rupture than protein-free lipid-based LUVs. ${ }^{17}$

To further characterize and validate this method, the glutamate sensor was exposed to LUVs encapsulating 5 different concentrations of glutamate $(100,150$, 200, 250 and $300 \mathrm{mM}$ ). The LUVs were prepared by a gentle swelling method, using five rounds of freeze-thaw cycles to encapsulate glutamate concentration and multiple extrusion passages to ensure a homogenous LUV size (Figure 2C). ${ }^{20,21}$ Through altering the applied potential from $-0.5 \mathrm{~V}$ to $-0.7 \mathrm{~V}$ and $-0.9 \mathrm{~V}$, we observed vesicles rupturing at the sensor surface in a potential dependent manner. The average frequency of vesicles bursting was significantly increasing with potential amplitude (Figure 2B), whereas for each potential applied, the average frequency remained constant over the time course for measurements (Figure 2A). It was also noted that the concentration of the encapsulating glutamate solution, regardless of the potential applied, could enhance likelihood of vesicle shattering, possibly as a result of glutamate interactions with the vesicle membrane, which may alter the vesicle membrane elastic properties and favor membrane rupture (Table S1). ${ }^{22-28}$ These observations suggest that the electric field near the sensor surface induces membrane pore formation at vesicles in close contact with the sensor surface, which is in agreement with previous observations for analysis of catecholamine vesicles when applying potentials of opposite polarity. ${ }^{14,15}$ 
A common issue with amperometric enzyme-based sensors, is that the detection efficiency is often less then $100 \%$, and therefore appropriate calibration methods are needed for quantitative analysis. Previous characterization of our ultrafast glutamate sensor demonstrated a linear concentration range between 10-100 $\mu \mathrm{M}$, as determined by steady state current amplitudes in bulk solution system. ${ }^{19}$ However, the experimental conditions for this new method analyzing glutamate content in synaptic vesicle differ significantly from saturated sensor surface measurements. In these measurements we only detect sub-millisecond events of nanosized vesicles emptying onto the surface of a 33-um (in diameter) sensor their $20 \mathrm{zL}$ internal solution containing on the order of only a few thousands of neurotransmitter molecules. ${ }^{8,29}$ We therefore designed a calibration system that mimics the experimental conditions used for synaptic vesicle analysis. This was realized by preparation of LUVs pre-filled with various physiologically relevant concentrations of glutamate solution and performing analysis of them using our ultrafast glutamate sensor. By plotting the charge density for each current spike detected (Q/LUV volume) versus the glutamate concentration used to load the LUVs, we found a linear relationship that was valid for quantitative glutamate analysis of single synaptic vesicle (Figure 2D). Although neither the applied potential nor the encapsulated glutamate concentration affected the rupture kinetics (Figure S1, Table S2), we noted that Q and the current spike maximal amplitude $\left(I_{\max }\right)$ were amplified with the increasing applied potential, which is in agreement with sensor voltammograms for $\mathrm{H}_{2} \mathrm{O}_{2} \cdot{ }^{19,30}$ However, for each constant potential applied, linear calibration graphs were achieved (Figure 2D and S2), validating the LUV calibration method for quantitative glutamate analysis of single synaptic vesicles.
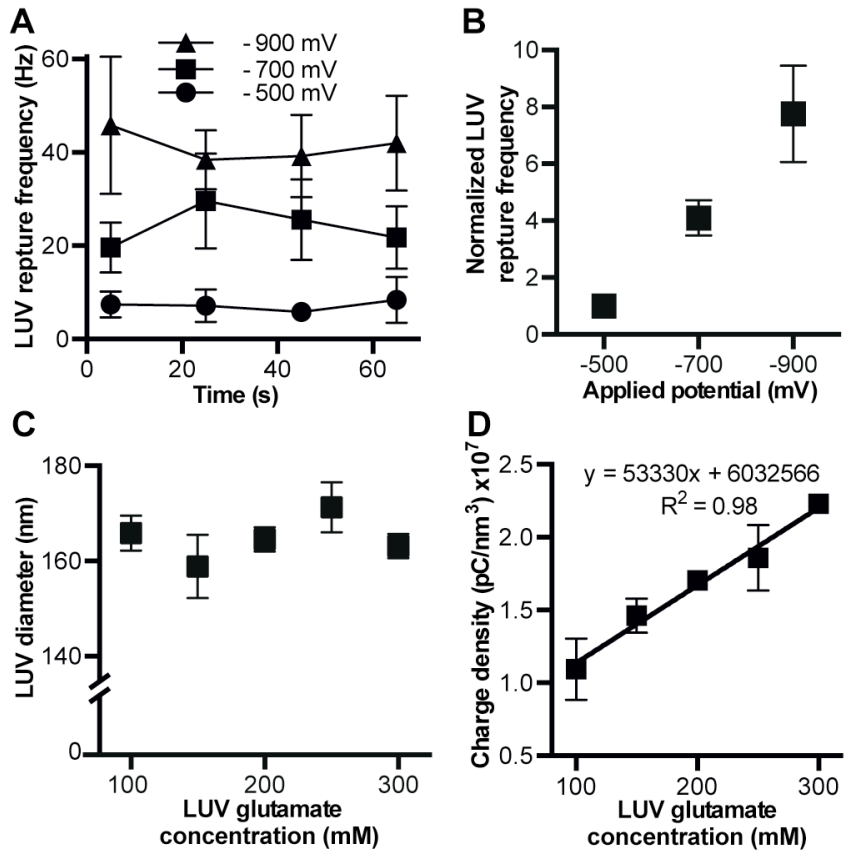

Figure 2. Characterization of synaptic vesicle glutamate analysis method by amperometrically detecting rupture of LUVs loaded with 5 different glutamate concentrations. (A) The average frequency of LUV rupture versus time when applying a $-0.5,-0.7$ or $-0.9 \mathrm{~V}$ potential. Error bars are standard deviation of the mean (SEM). (B) The average frequency of LUV rupture versus the applied sensor potential. Each data point was averaged over the initial $65 \mathrm{~s}$ of recording and normalized to the frequency recorded at $-0.5 \mathrm{~V}$. Error bars are SEM. (C) The average LUV size versus concentration of the encapsulating glutamate solution $(n=3)$, measured by nanosized tracking analysis system. Error bars are standard deviation. (D) Calibration curve constructed from the average charge detected from vesicle glutamate release $(n=4-5)$ versus the LUV glutamate concentration. Error bars are SEM.

To determine the average number of glutamate molecules stored inside of single synaptic vesicles, quantitative measurements using the ultrafast glutamate sensor was performed immediately after isolation of synaptic vesicles that was performed in the presence of ATP to minimize glutamate leakage before analysis. Assuming a vesicle internal volume of $20 \mathrm{zL}$, these measurements showed that an average of $8300 \pm 600$ glutamate molecules are stored in each synaptic vesicle and corresponds to a concentration of $\sim 0.7 \pm$ 
$0.05 \mathrm{M}$ glutamate. We note that this is a magnitude of approximately 3-10 times larger concentration than previously stated for this neurotransmitter that has been so difficult to quantify. ${ }^{10,11}$ This is in line with recent estimations using postsynaptic receptor saturation measurement and computer modeling of quantal release. ${ }^{33}$ This result could be related to that our newly developed ultrafast glutamate sensor allows direct measurement of glutamate at single synaptic vesicle level and perhaps also that we might have used a synaptic vesicle protocol that better retain the vesicle glutamate content before analysis. Interestingly, this neurotransmitter concentration then matches the concentrations previously measured for catecholamine containing vesicles. $^{31}$

These experimental conditions also resemble those for amperometric recording of exocytosis at secretory cells, where typically a microelectrode is placed in close proximity to a cell surface to capture isolated current spikes from single vesicle neurotransmitter release events. Therefore, we also applied the LUV-based calibration to our recordings of spontaneous glutamate in the core region of the nucleus accumbens of mouse (Figure 3A) and rat (Figure S3B) brain slices. Analysis of the sub-millisecond kinetics (Table S3 and S4) and the charge, Q, for each category of spike detected in brain tissue, and the average number of glutamate molecules released from exocytotic events was determined. Except for "funky spikes" that we believe may represent multiple vesicle detection and "overlap" which might represent codetection, all other categories of spikes were analyzed according to their shape and assumed to represent single exocytosis events. ${ }^{19}$ This analysis revealed that about $5200 \pm 850$ (average of means \pm SEM) glutamate molecules were released when sharp spikes, which were the most frequently found spikes (50\%), were detected. ${ }^{19}$ While an average of $8500 \pm$ 1100 glutamate molecules were measured at events that can be characterized as more complex plateau shaped spikes (Figure 3A). Assuming that a full exocytosis model dominates these recordings, , this predicts an original vesicular glutamate concentration of $0.7 \pm 0.05 \mathrm{M}$. By comparing the measured exocytotic glutamate release with the number of glutamate molecules stored in the isolated synaptic vesicles, we note that only part of the glutamate content is detected when monitoring sharp current spikes, whereas the more complex current spikes seem to represent that the entire vesicle glutamate content is being emptied (Figure 3B). Similar variability in current spike dynamics were previously described by amperometric dopamine recording from neurons of the rat ventral tegmental area and suggested to represent different modes of exocytosis as well as multivesicle release events. Sharp spikes were denoted by less dopamine detection and more complex shaped spikes represented a larger number of dopamine molecules released per vesicle..$^{32}$ However, more work is needed to characterize the mechanisms that are responsible for the different current spike dynamics observed in brain tissue, as variations in quantal release might be a result of glutamate release from different cell types or different modes of exocytosis.

The experiments shown here present a methodology to count the number of glutamate molecules loaded inside single isolated synaptic vesicles and provide new means to quantify the amount glutamate released by single exocytosis events in brain tissue during neuronal communication. We found that by counting the number of glutamate molecules stored inside isolated synaptic vesicles and determining the number of glutamate molecules released at exocytosis, the glutamate concentrations are similar to concentrations of catecholamines previously found in catecholaminecontaining vesicles. Collectively, this novel method offers new means to study the dynamics of glutamate vesicle loading, storage and regulatory aspects of synaptic glutamate transmission that can help to gain a clearer view of mechanisms of the glutamate system involved in neurotransmission of both healthy and disease states. 

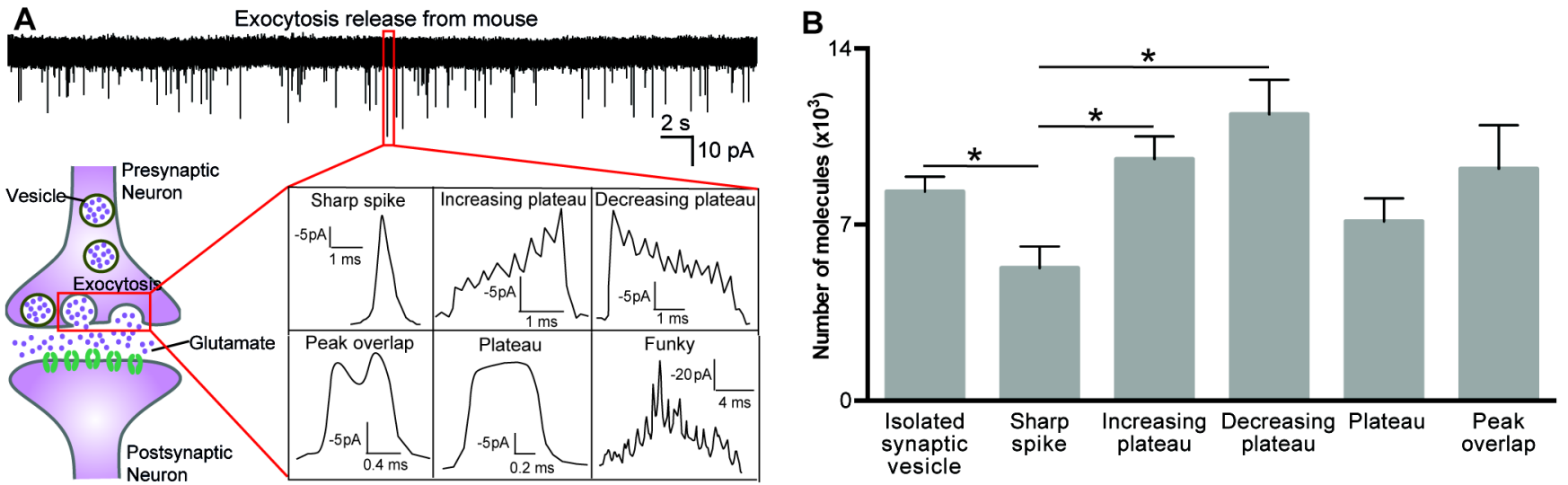

Figure 3. (A) A representative amperometric recordings $(10 \mathrm{kHz})$ of exocytotic glutamate release events in the core region of the nucleus accumbens of mouse brain slice $(n=4)$ with an illustration of the 6 different current spike types associated with glutamate release in rodent brain tissue. Schematic is not drawn to scale. (B) Comparison of the average number of glutamate molecules stored in synaptic vesicles isolated from mouse brain $(n=4)$ and the average number of glutamate molecules released by exocytosis in the mouse brain slice $(n=4)$ and as by the 5 different categories of current spike shapes detected. All amperometric data was collected at a $-0.5 \mathrm{~V}$ potential vs $\mathrm{Ag} / \mathrm{AgCl}$ reference electrode. Data is presented as average of the means \pm SEM. Two-tailed paired student's t-test was performed to compare the glutamate number, $* p<0.05$.

\section{ASSOCIATED CONTENT}

Supporting Information. Additional data including lipid vesicle current spike kinetics, frequency of vesicle rupture at different applied potential with time, kinetics of amperometric spike in rat brain, and methods in experimental section.

\section{AUTHOR INFORMATION}

\section{Corresponding Author}

* cans@chalmers.se

\section{Funding Sources}

We appreciate financial support from The Swedish Research Council, The Swedish Brain Foundation, Ragnar Söderberg Foundation, The Novo Nordisk Foundation, The Wallenberg Center for Molecular and Translational Medicine at the University of Gothenburg, Ernst and Fru Rådman Colliander Stiftelse, Wilhelm and Martina Lundgren Stiftelse and Magnus Bergvall Stiftelse.

\section{Notes}

The authors declare no competing financial interest.

\section{ACKNOWLEDGMENT}

We thank Dr. Eric Hanse for the use of microscope and low-current potentiostat.

\section{REFERENCES}

(1) Zhou, Y.; Danbolt, N. C. Glutamate as a Neurotransmitter in the Healthy Brain. J. Neural Transm. 2014, 121, 799-817.

(2) Hyman, S. E.; Malenka, R. C.; Nestler, E. J. NEURAL MECHANISMS OF ADDICTION: The Role of Reward-Related Learning and Memory. Annu. Rev. Neurosci. 2006, 29, 565-598.

(3) Takamori, S. Presynaptic Molecular Determinants of Quantal Size. Front. Synaptic Neurosci. 2016, 8.

(4) Sulzer, D.; Cragg, S. J.; Rice, M. E. Striatal Dopamine Neurotransmission: Regulation of Release and Uptake. Basal Ganglia 2016, 6, 123-148.

(5) McEntee, W. J.; Crook, T. H. Glutamate: Its Role in Learning, Memory, and the Aging Brain. Psychopharmacology (Berl). 1993, 111, 391-401.

(6) Meldrum, B. S. Glutamate as a Neurotransmitter in the Brain: Review of Physiology and Pathology. J. Nutr. 2000, 130, 1007S-1015S.

(7) Djuricic, B. Glutamate in brain: transmitter and poison. Glas. Srp. Akad. Nauka Med. 2002, No. 47, 55-76.

(8) Takamori, S.; Holt, M.; Stenius, K.; Lemke, E. A.; Grønborg, M.; Riedel, D.; Urlaub, H.; Schenck, S.; Brügger, B.; Ringler, P.; et al. Molecular Anatomy of a Trafficking Organelle. Cell 2006, 127, 831-846.

(9) Budzinski, K. L.; Allen, R. W.; Fujimoto, B. S.; Kensel-Hammes, P.; Belnap, D. M.; Bajjalieh, S. M.; Chiu, D. T. Large Structural Change in Isolated Synaptic Vesicles upon Loading with Neurotransmitter. Biophys. J. 2009, 97, 2577-2584.

(10) Riveros, N.; Fiedler, J.; Lagos, N.; Mun oz, C.; Orrego, F. Glutamate in Rat Brain Cortex Synaptic 
Vesicles: Influence of the Vesicle Isolation Procedure. Brain Res. 1986, 386, 405-408.

(11) Burger, P. M.; Mehl, E.; Cameron, P. L.; Maycox, P. R.; Baumert, M.; Lottspeich, F.; De Camilli, P.; Jahn, R. Synaptic Vesicles Immunoisolated from Rat Cerebral Cortex Contain High Levels of Glutamate. Neuron 1989, 3, 715-720.

(12) Budzinski, K. L.; Zeigler, M.; Fujimoto, B. S.; Bajjalieh, S. M.; Chiu, D. T. Measurements of the Acidification Kinetics of Single SynaptopHluorin Vesicles. Biophys. J. 2011, 101, 1580-1589.

(13) Farsi, Z.; Preobraschenski, J.; van den Bogaart, G.; Riedel, D.; Jahn, R.; Woehler, A. Single-Vesicle Imaging Reveals Different Transport Mechanisms between Glutamatergic and GABAergic Vesicles. Science (80-. ). 2016, 351, 981-984.

(14) Omiatek, D. M.; Bressler, A. J.; Cans, A. S.; Andrews, A. M.; Heien, M. L.; Ewing, A. G. The Real Catecholamine Content of Secretory Vesicles in the CNS Revealed by Electrochemical Cytometry. Sci. Rep. 2013, 3, 1-6.

(15) Dunevall, J.; Fathali, H.; Najafinobar, N.; Lovric, J.; Wigström, J.; Cans, A.-S.; Ewing, A. G. Characterizing the Catecholamine Content of Single Mammalian Vesicles by Collision-Adsorption Events at an Electrode. J. Am. Chem. Soc. 2015, 137, 43444346.

(16) Hochstetler, S. E.; Puopolo, M.; Gustincich, S.; Raviola, E.; Wightman, R. M. Real-Time Amperometric Measurements of Zeptomole Quantities of Dopamine Released from Neurons. Anal. Chem. 2000, 72, 489496.

(17) Lovrić, J.; Najafinobar, N.; Dunevall, J.; Majdi, S.; Svir, I.; Oleinick, A.; Amatore, C.; Ewing, A. G. On the Mechanism of Electrochemical Vesicle Cytometry: Chromaffin Cell Vesicles and Liposomes. Faraday Discuss. 2016, 193, 65-79.

(18) Day, B. K.; Pomerleau, F.; Burmeister, J. J.; Huettl, P.; Gerhardt, G. A. Microelectrode Array Studies of Basal and Potassium-Evoked Release of IGlutamate in the Anesthetized Rat Brain. J. Neurochem. 2006, 96, 1626-1635.

(19) Wang, Y.; Mishra, D.; Bergman, J.; Keighron, J. D.; Skibicka, K. P.; Cans, A.-S. Ultrafast Glutamate Biosensor Recordings in Brain Slices Reveal Complex Single Exocytosis Transients. ACS Chem. Neurosci. 2019, 10, 1744-1752.

(20) Simonsson, L.; Kurczy, M. E.; Trouillon, R.; Hook, F.; Cans, A.-S. A Functioning Artificial Secretory Cell. Sci. Rep. 2012, 2, 824.

(21) Mayer, L. D.; Hope, M. J.; Cullis, P. R.; Janoff, A. S. Solute Distributions and Trapping Efficiencies
Observed in Freeze-Thawed Multilamellar Vesicles. Biochim. Biophys. Acta - Biomembr. 1985, 817, 193196.

(22) Wang, C.; Ye, F.; Velardez, G. F.; Peters, G. H.; Westh, P. Affinity of Four Polar Neurotransmitters for Lipid Bilayer Membranes. J. Phys. Chem. B 2011, 115, 196-203.

(23) Tsong, T. Y. Electroporation of Cell Membranes. Biophys. J. 1991, 60, 297-306.

(24) Lipowsky, R. Bending of Membranes by Anchored Polymers. Europhys. Lett. 1995, 30, 197202.

(25) Simon, J.; Kühner, $M_{\text {.; }}$ Ringsdorf, $H_{\text {.; }}$; Sackmanna, E. Polymer-Induced Shape Changes and Capping in Giant Liposomes. Chem. Phys. Lipids 1995, 76, 241-258.

(26) Döbereiner, H.-G.; Selchow, O.; Lipowsky, R. Spontaneous Curvature of Fluid Vesicles Induced by Trans-Bilayer Sugar Asymmetry. Eur. Biophys. J. 1999, 28, 174-178.

(27) Kakorin S. Neumann, E. Kinetics of the Electroporative Deformation of Lipid Vesicles and Biological Cells in an Electric Field. Berichte der Bunsengesellschaft für Phys. Chemie 1998, 102, 670-675.

(28) Reimhult, E.; Höök, F.; Kasemo, B. Intact Vesicle Adsorption and Supported Biomembrane Formation from Vesicles in Solution: Influence of Surface Chemistry, Vesicle Size, Temperature, and Osmotic Pressure. Langmuir 2003, 19, 1681-1691.

(29) Newton, A. J. H.; Wall, M. J.; Richardson, M. J. E. Modeling Microelectrode Biosensors: Free-Flow Calibration Can Substantially Underestimate Tissue Concentrations. J. Neurophysiol. 2017, 117, 937-949.

(30) Keighron, J. D.; Wigström, J.; Kurczy, M. E.; Bergman, J.; Wang, Y.; Cans, A.-S. Amperometric Detection of Single Vesicle Acetylcholine Release Events from an Artificial Cell. ACS Chem. Neurosci. 2015, 6, 181-188.

(31) Bruns, D.; Jahn, R. Real-Time Measurement of Transmitter Release from Single Synaptic Vesicles. Nature 1995, 377, 62-65.

(32) Staal, R. G. W.; Mosharov, E. V.; Sulzer, D. Dopamine Neurons Release Transmitter via a Flickering Fusion Pore. Nat. Neurosci. 2004, 7, 341346.

(33) Budisantoso, T.; Harada, H.; Kamasawa, N.; Fukazawa, Y.; Shigemoto, R.; Matsui, K. Evaluation of Glutamate Concentration Transient in the Synaptic Cleft of the Rat Calyx of Held. J. Physiol. 2013, 591, 219-239. 
Table of Contents

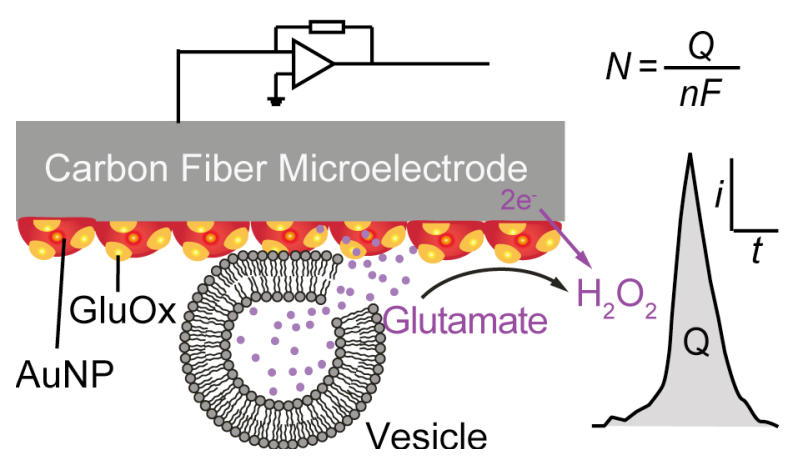




\section{Counting the Number of Glutamate Molecules in Single Synaptic Vesicles}

Yuanmo Wang, ${ }^{\dagger}$ Hoda Fathali, ${ }^{\dagger}$ Devesh Mishra, ${ }^{\neq, \perp}$ Thomas Olsson,,$"$ Jacqueline D. Keighron", Karolina P. Skibicka, ${ }^{\perp, \neq}$ and Ann-Sofie Cans*,t

${ }^{\dagger}$ Department of Chemistry and Chemical Engineering, Chalmers University of Technology, Kemigården 4, SE-412 96 Gothenburg, Sweden;

'Department of Physiology/Metabolic Physiology, Institute of Neuroscience and Physiology, The Sahlgrenska Academy at the University of Gothenburg, Medicinaregatan 11, SE-413 90 Gothenburg, Sweden;

"Department of Chemical and Biological Sciences, New York Institute of Technology, Old Westbury, NY 11568, USA

"Department of Physics, Chalmers University of Technology, Kemigården 4, SE-412 96 Gothenburg, Sweden;

${ }^{\perp}$ Wallenberg Centre for Molecular and Translational Medicine, University of Gothenburg, SE-405 30 Gothenburg, Sweden

Corresponding author email address:

*cans@chalmers.se

\section{Table of Content}

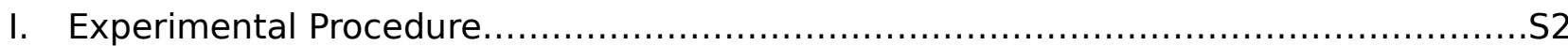

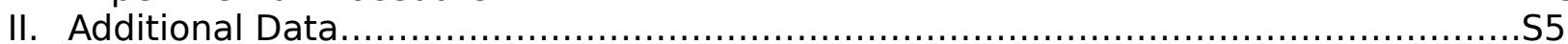

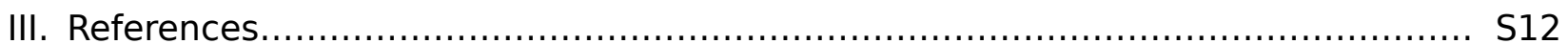




\section{Experimental Procedure}

Materials. 5U L-Glutamate Oxidase (GluOx) lyophilized powder from Streptomyces sp., sodium chloride ( $\mathrm{NaCl}$ ), sodium bicarbonate $\left(\mathrm{NaHCO}_{3}\right)$, gold chloride trihydrate $\left(\mathrm{HAuCl}_{4}\right)$, ferrocene-methanol $(\mathrm{FcMeOH})$, Whatman ${ }^{\circledR}$ Anotop ${ }^{\circledR} 25$ syringe filters with $20 \mathrm{~nm}$ filtering size, phosphate-buffered saline (PBS) tablets (10 mM, pH 7.2), sulfuric acid $\left(\mathrm{H}_{2} \mathrm{SO}_{4}\right)$, copper sulfate $\left(\mathrm{CuSO}_{4}\right)$, monosodium glutamate, glycerol, potassium chloride $(\mathrm{KCl})$, monosodium phosphate (anhydrous, $\left.\mathrm{NaH}_{2} \mathrm{PO}_{4}\right)$, calcium chloride $\left(\mathrm{CaCl}_{2}\right)$, magnesium chloride $\left(\mathrm{MgCl}_{2}\right)$, magnesium sulfate $\left(\mathrm{MgSO}_{4}\right)$, Adenosine 5'-triphosphate (ATP), cOmplete (EDTA-free) tablets, glucose, tungsten wire, HEPES and sucrose were purchased from Sigma-Aldrich (St. Louis, MO, USA). 1,2-dioleoyl-sn-glycero-3phosphocholine (DOPC), 1,2-dioleoyl-sn-glycero-3-phosphoethanolamine (DOPE) and cholesterol were purchased from Avanti Polar Lipids Inc., USA. MilliQ water with resistivity $\geq 18 \mathrm{M} \Omega . c m$ was used in all experiments.

Preparation of glutamate-filled LUVs. Lipid vesicles were prepared by mixing DOPC, DOPE and cholesterol in mass ratio 50:25:25 to achieve molar ratio 39:21:40. The flask containing mixed lipids was rotoevaporated under $\mathrm{N}_{2}$ for three hours to eliminate the chloroform used for dissolving the lipids and to form a thin lipid film at the surface of the glass rotoevaporation flask at vacuum condition. The lipid vesicles were formed by rehydrating the thin lipid film with glutamate buffer solution during an incubation of $30 \mathrm{~min}$ at room temperature. The total lipid concentrations were approximate $2.5 \mathrm{mg} / \mathrm{ml}$ after the dehydration process. To entrap a homogenous glutamate solution inside the lipid vesicles, Falcon tubes containing rehydrated lipid vesicle solution was alternately placed into liquid nitrogen and room temperature water to create five freeze-thaw cycles. ${ }^{15}$ To unify the vesicle size, the lipid vesicle suspension was extruded through a $400 \mathrm{~nm}$ pore sized polycarbonate membrane (Whatman, USA) 21 times using Avanti Mini-Extruder (Avanti Polar Lipids, Inc., USA) under the same pressure (i.e. 1 bar) generated by $\mathrm{N}_{2}$ gas at room temperature. Illustra ${ }^{\mathrm{TM}}$ Microspin $^{\mathrm{TM}} \mathrm{S}-200 \mathrm{HR}$ columns (GE Healthcare, Buckinghamshire, UK.) were used to remove the non-loaded glutamate from the vesicle suspension. The collected glutamate lipid vesicle solution was used immediately in experiments within 4 hours of synthesis and stored at $4{ }^{\circ} \mathrm{C}$ during waiting time.

Glutamate loading buffer were prepared by dissolving monosodium glutamate into $10 \mathrm{mM}$ HEPES with $\mathrm{pH} 7.4$ at different concentrations as follows: $100 \mathrm{mM}$ with osmotic pressure 156 $\mathrm{mOsm} / \mathrm{kg}, 150 \mathrm{mM}$ with $262 \mathrm{mOsm} / \mathrm{kg}, 200 \mathrm{mM}$ with $343 \mathrm{mOsm} / \mathrm{kg}, 250 \mathrm{mM}$ with 421 $\mathrm{mOsm} / \mathrm{kg}$ and $300 \mathrm{mM}$ with $496 \mathrm{mOsm} / \mathrm{kg}$. The dilution buffer needed for electrochemical and vesicle size measurements were made by dissolving $\mathrm{NaCl}$ into $10 \mathrm{mM}$ HEPES with $\mathrm{pH} 7.4$, the osmotic pressure of dilution solution in $10 \mathrm{mM}$ HEPES ( $\mathrm{pH} 7.4$ ) was adjusted by $\mathrm{NaCl}$ to a same value that matches the corresponding glutamate concentration that was used for loading the vesicles.

Size measurements of glutamate of LUVs. The size distribution of glutamate lipid vesicles was measured using Nanoparticle Tracking Analysis (NTA) system (NanoSight LM10, Malvern Instruments Ltd, UK). For each vesicle batch the sample was first run at $2000 \times$ dilution using the osmotic pressure-controlled dilution buffer to determine the vesicle concentration; based on the outcome, the sample dilution was then adjusted to match the optimal concentration for measurement by the instrument. All measurements were done at a constant temperature (25 ${ }^{\circ} \mathrm{C}$ ) using a module and for each sample, five measurements were recorded for a period of 1 minute, during flow condition (to increase number of particles tracked). All size measurements were carried out immediately after lipid vesicles preparation.

Fabrication of an ultrafast glutamate sensor. Carbon fiber microelectrodes were made by aspirating single $33 \mu \mathrm{m}$ in diameter carbon fibers into borosilicate glass capillaries (1.2 $\mathrm{mm}$ outer diameter, $0.69 \mathrm{~mm}$ inner diameter, Sutter Instrument Co., Novato, CA, USA). The capillaries with a carbon fiber inserted on the inside were then pulled from the using a 
micropipette puller (model P-1000, Sutter Instrument Co., Novato, USA) to created two tapered glass-coated carbon fiber tip electrodes. To seal the glass-carbon fiber junction of the electrode, the electrode tips were placed into epoxy solutions (EpoTek 301, Epoxy Technology, Billerica, MA, USA) for 2 minutes and thereafter incubated in the oven at $100{ }^{\circ} \mathrm{C}$ overnight. The electrode tips were then cut near the carbon-glass junction under microscope using a scalpel and were thereafter polished at a $45^{\circ}$ angle using a micropipette beveller (model BV10, Sutter Instrument Co., Novato, USA) to achieve a glass-coated insulated disc electrode surface. Prior to further modifying the electrode tip surface, the electrodes were backfilled with $3 \mathrm{M} \mathrm{KCl}$ solution and a tungsten wire were used to connect the electrode to a an electrical head stage to test the electrode performance in a $1 \mathrm{mM} \mathrm{FCMeOH}$ solution by performing cyclic voltammetry and scanning the voltage between $-0.2 \mathrm{~V}$ and $+0.8 \mathrm{~V}$ against a saturated $\mathrm{Ag} / \mathrm{AgCl}$ reference electrode ( $\mathrm{CH}$ Instruments, USA) at $0.1 \mathrm{Vs}^{-1}$ using a potentiostat (model 650A Series Multi-Potentiostat, $\mathrm{CH}$ Instruments, USA). Only electrodes with a stable steady-state current and expected current size amplitude were used.

Gold nanoparticle (AuNP) hemispheres were electrochemically deposited onto the electrode tip surface in a similar way to the method of Finot et. al. ${ }^{16}$ with minor alterations as performed in our lab ${ }^{12}$. Briefly here, the carbon working electrode and an $\mathrm{Ag} / \mathrm{AgCl}$ reference electrode were immersed into $0.5 \mathrm{mM} \mathrm{HAuCl}_{4}$ solution, a potential of $+1.2 \mathrm{~V}$ was applied to the carbon electrode surface for $10 \mathrm{~s}$ followed by switching the potential down to $-0.6 \mathrm{~V}$ for $24 \mathrm{~s}$ via the 650A Series Multi-Potentiostat (CH Instruments, USA). The AuNP surface area was examined electrochemically by applying potential onto the AuNP-coated carbon electrode against a $\mathrm{Cu} / \mathrm{CuSO}_{4}$ reference electrode via a linear sweep from $+1.4 \mathrm{~V}$ (held for $5 \mathrm{~s}$ ) to $+0.5 \mathrm{~V}$ at a rate of $0.1 \mathrm{Vs}^{-1}$ in $500 \mathrm{mM} \mathrm{H}_{2} \mathrm{SO}_{4}{ }^{12}$. The charge of deposited AuNP was determined by integrating the resulting peak at around $+0.8 \mathrm{~V}$ and the obtained charge was divided with a constant derived from the Finot method that was equal to $489 \mu \mathrm{C} \mathrm{cm}^{-2} \cdot{ }^{16}$

At the last step of the glutamate sensor fabrication, an ultra-thin layer of enzyme was immobilized onto the AuNP-coated carbon electrode using the protocol developed from our lab that control the enzyme layer thickness to a monolayer. ${ }^{11}$ To completely dissolve the enzymes used for coating the electrode, $500 \mu \mathrm{L}$ of $10 \mathrm{mM}$ sodium bicarbonate ( $\mathrm{pH}$ 8.2) was added into the L-Glutamate Oxidase lyophilized powder bottle $(5 \mathrm{U})$ and allowed the enzyme to dissolve by gently rotate the bottle. GluOx was allowed to self-adsorb onto the sensor surface by immersing the AuNP-coated electrode tip into $100 \mu \mathrm{l}$ freshly prepared enzyme solution for approximate 2-3 hours at room temperature. The glutamate sensors were immediately used after fabrication, or otherwise stored in $10 \mathrm{mM}$ PBS (pH 7.2) at $4{ }^{\circ} \mathrm{C}$ before use within 6 hours.

Mouse and rat brain slice preparation. Rodent brain slices were prepared from the brain of 4 - 8 weeks old male mice (C57BL) and rats (Sprague Dawley) that were purchased from Charles River, Sulzfeld, German. The rodents were group housed in a 12-h light/dark cycle with 0700 hours light on and supplied with ad libitum chow (Teklad Global 16\% Protein Rodent Diet 2016, Envigo, Huntingdon, UK) and water. The animal experiments were performed in accordance with the Swedish Animal Welfare law and the local ethical committee regulations for animal research with ethics number 29-2014 at the University of Gothenburg, Sweden. Before decapitation, they were anesthetized using isoflurane (Baxter Medical AB, Sweden), brains were quickly removed and cut into $400 \mu \mathrm{m}$ slices using Microtome HM $650 \mathrm{~V}$ (Thermo Fisher Scientific, Loughborough, UK) in $3{ }^{\circ} \mathrm{C}$ glycerol solution containing: $11 \mathrm{mM}$ glucose, $7 \mathrm{mM}$ $\mathrm{MgCl}_{2}, 1.2 \mathrm{mM} \mathrm{CaCl}, 2.5 \mathrm{mM} \mathrm{KCl}, 25 \mathrm{mM} \mathrm{NaHCO}_{3}, 1.2 \mathrm{mM} \mathrm{NaH} \mathrm{PO}_{4}$ and $219 \mathrm{mM}$ glycerol. Coronal slices containing nucleus accumbens were incubated in medium containing $15 \mathrm{mM}$ sucrose, $4 \mathrm{mM} \mathrm{MgCl}$, $10 \mathrm{mM}$ D-Glucose, $1 \mathrm{mM} \mathrm{CaCl}, 25 \mathrm{mM} \mathrm{NaHCO}, 1.25 \mathrm{mM} \mathrm{NaH}_{2} \mathrm{PO}_{4}, 2.5$ $\mathrm{mM} \mathrm{KCl}, 125 \mathrm{mM} \mathrm{NaCl}$ for at least $45 \mathrm{~min}$ at $30{ }^{\circ} \mathrm{C}$, and then transferred into a microscopeassociated submerged chamber filled with artificial cerebrospinal fluid (aCSF) solution at a flow rate of $1 \mathrm{ml} \mathrm{min}^{-1}$ at room temperature. aCSF solution containing $2 \mathrm{mM} \mathrm{CaCl}, 25 \mathrm{mM} \mathrm{NaHCO}_{3}$, $2.5 \mathrm{mM} \mathrm{KCl}, 125 \mathrm{mM} \mathrm{NaCl}, 10 \mathrm{mM} \mathrm{D}$-Glucose 10 and $1.3 \mathrm{mM} \mathrm{MgCl}_{2}$ were aerated with mixture of $5 \%$ carbon dioxide and $95 \%$ oxygen for at least $30 \mathrm{~min}$ before putting in brain slice and aeration was continued during the whole recording process. 
Preparation of isolated synaptic vesicle. Synaptic vesicles were isolated from the same fresh mice brain that were used in the brain slice experiment. The work was performed with protocol that was summarized by the group of Jahn $\operatorname{lab}^{17}$ and the group of Chiu lab ${ }^{18}$ with slightly modification. Briefly, a whole brain was removed from one decapitated mouse and washed in ice-cold homogenization buffer containing $10 \mathrm{mM}$ HEPES, $300 \mathrm{mM}$ sucrose, $4 \mathrm{mM}$ $\mathrm{KCl}, 4 \mathrm{mM} \mathrm{MgSO}_{4}$ with freshly added $4 \mathrm{mM}$ ATP and protease inhibitors cOmplete, $\mathrm{pH}$ 7.4. The collected brain was homogenized in ice-cold homogenization buffer with 10 strokes at 900 r.p.m. using a glass-Teflon homogenizer, followed by centrifuging the homogenization mixture at $1000 \mathrm{~g}$ at $4{ }^{\circ} \mathrm{C}$ for $10 \mathrm{~min}$. The collected supernatant (S1) was continuously centrifuged at $15000 \mathrm{~g}$ and $4{ }^{\circ} \mathrm{C}$ for $15 \mathrm{~min}$, the supernatant (S2) in this round was taken out and stored on ice. The left pellet (P2) was homogenized at 2000 r.p.m. with three strokes after adding $9 \mathrm{ml}$ ice-cold homogenization buffer and centrifuged at $17000 \mathrm{~g}$ for $15 \mathrm{~min}$ at $4{ }^{\circ} \mathrm{C}$, the supernatant (LS1) was again collected and mixed with S2. The LS1/S2 mixture was centrifuged at $48000 \mathrm{~g}$ for $25 \mathrm{~min}$ at $4{ }^{\circ} \mathrm{C}$ and collected the supernatant (CS1) that contain isolated glutamate synaptic vesicles. All high-speed centrifugation was performed using an ultracentrifuge (L-80, Beckman Coulter Inc., Indianapolis, IN, USA). CS1 solution was used for later electrochemical measurements.

Electrochemical measurements of glutamate content in glutamate-filled lipid vesicles. Prior to amperometric measurements of glutamate in synthetically prepared glutamate-filled lipid vesicles, the functionality of each glutamate sensors was tested in 100 mM glutamate solution (dissolved in $10 \mathrm{mM}$ HEPES with $\mathrm{pH}$ 7.4) by performing cyclic voltammetry that scans the potential on glutamate sensor between $-0.2 \mathrm{~V}$ and $+0.8 \mathrm{~V}$ versus a chlorinated $\mathrm{Ag}$ wire as reference electrode at $0.1 \mathrm{Vs}-1$ using a HEKA patch clamp amplifier (EPC 10, HEKA elektronik, Lambrecht, Germany), only sensors that gave a response of a correct estimated current amplitude for the reduction of hydrogen peroxide, which serve as the reporter molecule for glutamate were used. The glutamate-filled lipid vesicles were diluted about 2 to 5 times with the same dilution solution used for NTA measurements before electrochemical measurements to ensure vesicle rupture to be initiated. The amperometric recordings were performed by holding potential onto sensor at $0 \mathrm{mV}$ for $15 \mathrm{~s}$ and switched down to a constant potential for a 3-5 min recording time (i.e. $-500 \mathrm{mV},-700 \mathrm{mV}$ and $-900 \mathrm{mV}$ ) versus a $\mathrm{Ag} / \mathrm{AgCl}$ reference electrode that was placed in the lipid vesicle solution using a patch clamp amplifier (Axopatch 200B, Molecule Devices, Sunnyvale, CA, USA) with a low-noise data acquisition system Axon ${ }^{\mathrm{TM}}$ Digidata ${ }^{\circledR}$ 1550B (Molecule Devices, Sunnyvale, CA, USA). The data were sampled at room temperature and at $20 \mathrm{kHz}$ with an internal low pass Bessel filter at 2 $\mathrm{kHz}$. The scaled output was set to $0.5 \mathrm{mV} / \mathrm{pA}$.

Electrochemical measurements of glutamate exocytotic activity in rodent brain slice. The glutamate sensors were tested by chronoamperometry before performing ex vivo amperometric recordings of spontaneous exocytosis activity, by placement into $100 \mathrm{mM}$ glutamate bulk solution (dissolved in aCSF) and applied on a constant potential of $0 \mathrm{~V}$ for $10 \mathrm{~s}$ followed by a potential switch to $-0.5 \mathrm{~V}$ for $1 \mathrm{~min}$ (versus a $\mathrm{Ag} / \mathrm{AgCl}$ disc reference electrode) using a patch clamp amplifier (MultiClamp 700B, Molecule Devices, Sunnyvale, CA, USA). Only sensors which provided a steady-steady state current amplitude corresponding to the glutamate concentrations in solution were used for experiments. With a $45^{\circ}$ beveled electrode angle, the sensor tip was placed at the NAc region of coronal planes that contain glutamatergic terminals from various brain regions with the assistance of an upright Nikon E600FN (Nikon Inc., Japan) fluorescence microscope mounted with $4 \times$ lens, and by placement of the sensor in the brain slice using methods as previously published. ${ }^{11}$ The amperometric recordings were carried out by applying a constant potential of $-0.5 \mathrm{~V}$ to the sensor surface versus a $\mathrm{Ag} / \mathrm{AgCl}$ disc reference electrode, using the same MultiClamp 700B. Except for two measurements that were recorded using a digitizer (model Digidata 1440A, Molecule Devices, Sunnyvale, CA, USA) at $10 \mathrm{kHz}$ and filtered at $4 \mathrm{kHz}$ using the internal low pass 4-pole Bessel filter, all the other measurements were sampled at $20 \mathrm{kHz}$ and filtered at $10 \mathrm{kHz}$. Scaled output factor was $0.5 \mathrm{mV} / \mathrm{pA}$. 
Electrochemical measurements of glutamate in isolated synaptic vesicles. Before performing amperometric measurements of isolated glutamate synaptic vesicles, the glutamate sensors were tested in the same way as when used in brain slice experiments. CS1 solution were diluted 10 times before measurements using homogenization buffer. Amperometric measurements were performed by holding $0 \mathrm{mV}$ onto sensor surface versus a $\mathrm{Ag} / \mathrm{AgCl}$ disc reference electrode for $10 \mathrm{~s}$, followed by lowering the potential to $-0.5 \mathrm{~V}$ for $5-10$ min using a patch clamp amplifier (MultiClamp 700B) at room temperature. Control experiments were performed similarly using pure homogenization buffer solution. All measurements were recorded at $20 \mathrm{kHz}$ and filtered at $10 \mathrm{kHz}$ using an internal low pass Bessel filter. Scaled output was adjusted to $0.5 \mathrm{mV} / \mathrm{pA}$ as well.

Analysis of amperometric data. All amperometric data were analyzed with IgorPro 6.37 software (WaveMetrics, Lake Oswego, OR, USA) using an Igor Procedure File for single spike events developed by David Sulzer's group ${ }^{19}$. The data were smoothed down to $5 \mathrm{kHz}$ (binomial sm.), spikes were the current spikes detected exceed 5 times the signal of the standard deviation of the derivative of background current noise with respect to time. All traces were carefully checked and false-positive spikes were manually removed. Only current traces containing more than 30 spikes were used in the analysis. Different current spike parameters were defined as follows: $\mathrm{T}_{\text {rise }}$ and $\mathrm{T}_{\text {fall }}$ are rise and fall time between $25 \%$ and $75 \%$ of a peak signal, $\mathrm{T}_{1 / 2}$ is the time of half maximum of a peak, $I_{\max }$ is the highest current of a peak, $\mathrm{Q}$ is the charge detected in each peak.

\section{Additional Data}

In this supportive information we present additional data from the process of characterizing this new amperometric method for quantitative analysis of glutamate content in single synaptic vesicles using an ultrafast enzyme-based glutamate sensor. In addition, we characterized this method for quantitative analysis of rapid transients of glutamate release at single exocytosis events in brain tissue.

\section{The applied potential and the LUV glutamate concentration affect vesicle rupture frequency.}

To better understand what drives vesicles to rupture at the glutamate sensor surface and to characterize the experimental conditions for valid quantitative glutamate measurement in these applications, we first investigated the effect of applied potential on vesicles rupture 
frequency at the glutamate sensor surface. For this, we prepared samples of LUVs that were pre-filled with various concentrations of glutamate solution (100, 150, 200, 250, $300 \mathrm{mM})$ and then used our ultrafast glutamate sensor to probe the LUV glutamate content, while varying the applied potential from -0.5 to $-0.7 \mathrm{~V}$ and $-0.9 \mathrm{~V}$ ( $\mathrm{vs}$ a $\mathrm{Ag} / \mathrm{AgCl}$ reference electrode) at the sensor surface.

From these recordings, the average frequency of vesicle rupture events was determined both as a function of the applied potential and versus the glutamate concentration used for loading the LUVs and was measured at time intervals of 5, 25, 45 and $65 \mathrm{~s}$ from the initial start of the amperometric recordings. As summarized in Table S1 A, B, the results from this analysis showed that the applied electric field at the sensor surface has a direct impact, where a larger amplitude of the negative potential applied enhances vesicle rupture. This is similar to previous observations for carbon fiber amperometry analysis of catecholamine-containing vesicles, where a larger positive potential trigger vesicles to more frequently rupture at the electrode surface. ${ }^{1}$ Prevailing theory states that by increasing the potential the higher energy of the electric field created at the electrode surface, destabilizes the membrane of the vesicles that are adsorbed at the electrode surface, as vesicles often deform in response to the applied electric field, which facilitates formation transient pore forming across the vesicle membrane. ${ }^{2}$ These membrane pores are not stable and may either close or that the pore dilates to a larger size, if an the pore line tension reach a sufficient high energetic cost that vesicles fully break open. ${ }^{3,4}$ Taken together with our experiments on characterizing glutamate-containing vesicles, shows that it is not a matter of polarity, but rather the magnitude of the applied potential that is key. This is in line with the mechanism for electroporation that a larger electric field assists likelihood of pore formation in lipid membranes. As the electric field quickly loses its strength from the surface and into the physiological sample solution, vesicles need to be in close proximity to the electrode surface for the membrane to respond to the applied potential. Hence, a crucial aspect for the method to be valid for quantification of glutamate-containing vesicles is the design of our glutamate sensor. In contrast to conventional enzyme-based glutamate sensors where thicker layers of GluOx usually are immobilized to the sensor surface, our sensor is constructed with an ultra-thin enzyme coating. The enzyme layer is limited to the thickness of an enzyme molecular monolayer, which means that vesicles may come up to a few nanometers away from the electrode surface considering an estimated diameter of GluOx is about $7 \mathrm{~nm}$, assumed by considering its molecular weight.

In addition to this finding, we found that the concentration of the encapsulating glutamate solution also played a role in facilitating vesicle rupture. For the 5 concentrations of glutamate tested, we found that a $200 \mathrm{mM}$ glutamate solution had a 2- to 7-fold larger vesicle rupture activity than LUVs encapsulating a lower $(100,150 \mathrm{mM})$ or higher $(250,300 \mathrm{mM})$ glutamate concentration while applying a constant potential to the electrode surface independent of the amplitude of applied potential (Table S1A,B). This suggests that the encapsulation of glutamate solution into the LUVs significantly affect the vesicle membrane properties and its physical response to the applied electric field. Glutamate is one of the neurotransmitters known to bind lipid membranes and is also thought to partition into membrane to interact with the lipid head groups. ${ }^{5}$ This molecular interaction that is mainly electrostatic involve interaction with the inner leaflet of the lipid bilayer, and the glutamate molecules can cause structural rearrangements of lipids membrane, which alters the density of lipid packing. This can significantly alter the vesicle membrane permeability and elastic properties which are factors known to affect both vesicle adsorption and electroporation efficiency and hence can modify the vesicle's ability to rupture at the sensor surface. ${ }^{3,5-10}$ 
Table S1 (A). Frequency $(\mathrm{Hz})$ of vesicle rupture as a function of the applied potential and concentration of glutamate solution used for loading the LUVs.

\begin{tabular}{|c|c|c|c|c|c|}
\hline \multirow{2}{*}{$\begin{array}{l}\text { Applied } \\
\text { potential } \\
(\mathrm{mV})\end{array}$} & \multirow{2}{*}{$\begin{array}{c}\text { Concentration of } \\
\text { glutamate loaded } \\
\text { (mM) }\end{array}$} & \multicolumn{4}{|c|}{ Time after initiating the amperometric measurement $(\mathrm{Hz})$} \\
\hline & & $5 s$ & 25s & 45s & 65s \\
\hline \multirow{5}{*}{-500} & $100(n=4)$ & $8.3 \pm 3.0$ & $4.9 \pm 3.5$ & $4.3 \pm 2.8$ & $2.7 \pm 0.8$ \\
\hline & $150(n=4)$ & $2.7 \pm 0.7$ & $5.0 \pm 0.6$ & $5.5 \pm 1.3$ & $5.9 \pm 1.2$ \\
\hline & $200(n=4)$ & $18.0 \pm 11.6$ & $21.3 \pm 5.4$ & $12.0 \pm 4.1$ & $27.8 \pm 7.5$ \\
\hline & $250(n=5)$ & $4.2 \pm 1.1$ & $2.2 \pm 0.8$ & $3.2 \pm 1.2$ & $3.4 \pm 1.0$ \\
\hline & $300(n=4)$ & $3.9 \pm 0.9$ & $2.9 \pm 0.4$ & $4.4 \pm 0.7$ & $2.2 \pm 1.0$ \\
\hline \multirow{5}{*}{-700} & $100(n=4)$ & $15.8 \pm 6.5$ & $15.9 \pm 8.1$ & $11.1 \pm 7.1$ & $12.9 \pm 3.6$ \\
\hline & $150(n=4)$ & $14.9 \pm 2.5$ & $22.8 \pm 4.3$ & $25.5 \pm 5.3$ & $18.0 \pm 4.1$ \\
\hline & $200(n=4)$ & $40.8 \pm 4.7$ & $70.3 \pm 9.6$ & $58.5 \pm 7.8$ & $47.5 \pm 3.9$ \\
\hline & $250(n=4)$ & $15.5 \pm 2.8$ & $19.0 \pm 2.2$ & $13.5 \pm 2.2$ & $18.9 \pm 1.9$ \\
\hline & $300(n=4)$ & $10.8 \pm 1.1$ & $20.4 \pm 4.4$ & $19 \pm 3.5$ & $10.9 \pm 3.4$ \\
\hline \multirow{5}{*}{-900} & $100(n=4)$ & $18.2 \pm 2.5$ & $22.5 \pm 1.7$ & $28.1 \pm 5.6$ & $26.7 \pm 1.8$ \\
\hline & $150(n=4)$ & $49.7 \pm 11.3$ & $35.6 \pm 13.3$ & $38.7 \pm 13.6$ & $43.8 \pm 13.2$ \\
\hline & $200(n=4)$ & $100.0 \pm 28.8$ & $60.5 \pm 11.2$ & $73.3 \pm 22.3$ & $80.0 \pm 15.0$ \\
\hline & $250(n=4)$ & $38.9 \pm 5.4$ & $40.9 \pm 8.3$ & $32.2 \pm 5.7$ & $36.4 \pm 8.9$ \\
\hline & $300(n=4)$ & $22.3 \pm 5.1$ & $30.6 \pm 7.6$ & $23.5 \pm 1.3$ & $22.7 \pm 3.2$ \\
\hline
\end{tabular}

The data is presented as the mean frequency \pm SEM.

Table S1 (B). Frequency $(\mathrm{Hz})$ of vesicle rupture as a function of applied potential and concentration of glutamate solution used for loading LUVs, where each frequency value represent the average frequency for the first $65 \mathrm{~s}$ of recording time and for each concentration of glutamate solution used to pre-fill the LUVs, as listed in Table S2 (A).

\begin{tabular}{|c|c|c|c|c|c|}
\hline \multirow{2}{*}{$\begin{array}{c}\text { Applied } \\
\text { potential (mV) }\end{array}$} & \multicolumn{5}{|c|}{ Concentration of glutamate loaded (mM) } \\
\hline & $100 \mathrm{mM}$ & $150 \mathrm{mM}$ & $200 \mathrm{mM}$ & $250 \mathrm{mM}$ & $300 \mathrm{mM}$ \\
\hline$-500(n=4)$ & $4.8 \pm 1.2$ & $4.8 \pm 0.7$ & $19.8 \pm 7.5$ & $2.7 \pm 0.5$ & $3.3 \pm 0.5$ \\
\hline$-700(n=4)$ & $13.9 \pm 6.0$ & $20.3 \pm 4.9$ & $54.3 \pm 4.6$ & $16.7 \pm 2.1$ & $15.3 \pm 2.5$ \\
\hline$-900(n=4)$ & $23.9 \pm 1.9$ & $42.0 \pm 10.2$ & $78.4 \pm 8.3$ & $37.1 \pm 6.1$ & $24.8 \pm 2.5$ \\
\hline
\end{tabular}

The data are shown as the average of frequency at 5 different loaded concentrations \pm SEM.

The mechanism for vesicle rupture and content release are not affected by the applied sensor potential or the concentration of the encapsulating glutamate solution. 
After we found that the applied potential and the encapsulated glutamate concentration affected the mechanism for vesicle glutamate release through mediated electroporation at the sensor surface, we also studied the kinetic parameters of the recorded current spikes that were collected from the recording of glutamate release from glutamate-filled LUVs. The parameters extracted from each recorded current spike are as defined in the schematic in Figure S1A, which demonstrates a typical amperometric current spike detected in these measurements. In this analysis, we first examined the parameters featuring the temporal components such as the spike rise time $\left(\mathrm{T}_{\text {rise }}\right)$ that is marked as the time it takes for the peak current to rise from $25 \%$ to $75 \%$ of its maximum amplitude $\left(I_{\max }\right)$, the spike fall-time $\left(T_{\text {fall }}\right)$ measuring the time for current drop from $75 \%$ to $25 \%$ of $I_{\max }$ and the current spike half-time $\left(T_{1 / 2}\right)$ defined as the width of the spike at half-maximum amplitude. Here, $T_{\text {rise }}$ is thought to be related to the detection of glutamate released from the time the initial pore that is formed due to electroporation and is large enough for glutamate to escape and the glutamate that continuously is released through a pore the continues to dilate and finally leads to shattering of the organelle and the full vesicle glutamate content is released. Finally, the parameter $\mathrm{T}_{\text {fall }}$ is thought to be connected to the time that it takes for neurotransmitters to diffuse from the release site to the electrode surface for detection and $T_{1 / 2}$ is the timing of the event. As shown in Figure S1 B-D, it was evident that the spike kinetic parameters $T_{1 / 2}, T_{\text {rise }}$ and $T_{\text {fall }}$ were not affected by either the amplitude of the applied potential or the glutamate concentration encapsulated inside the LUVs. This suggests that once the initiate pore has been formed in vesicle membrane, it is the same mechanisms affecting the vesicle pore to dilate and rupture the vesicle, without significantly affecting the rate of the pore dilation and glutamate release from the vesicle compartment or its rate for detection at the electrode surface.

A

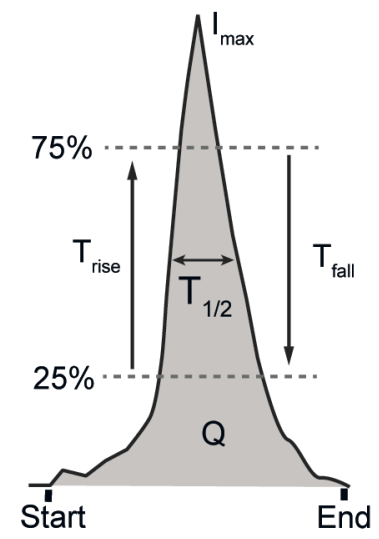

D

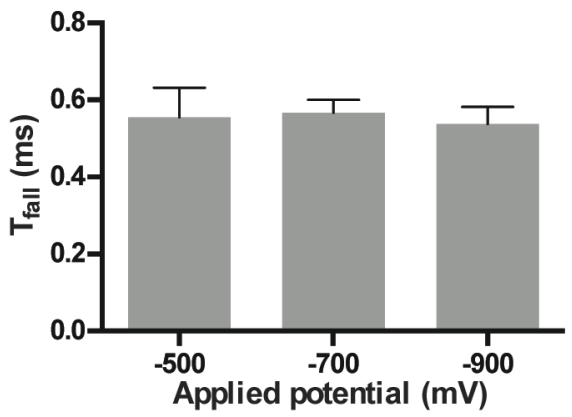

B

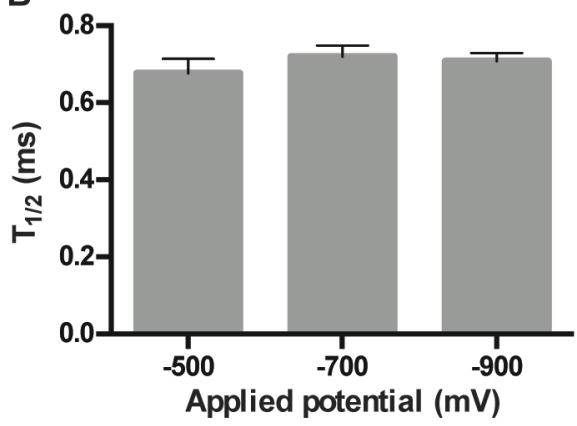

E

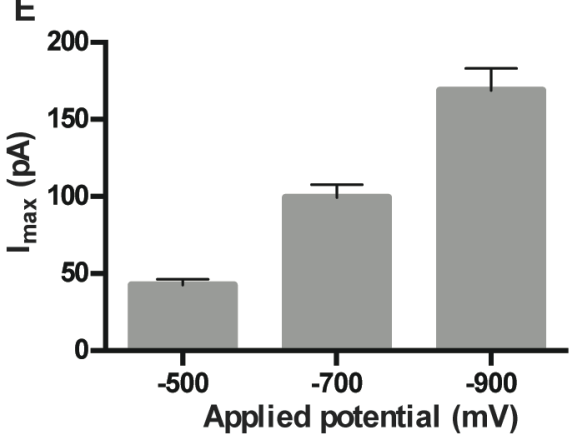

C

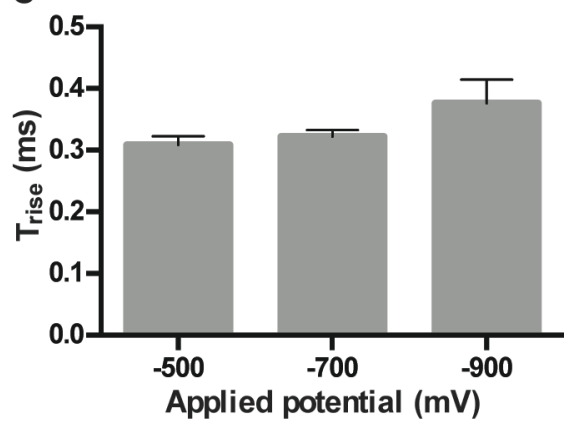

$\mathbf{F}$

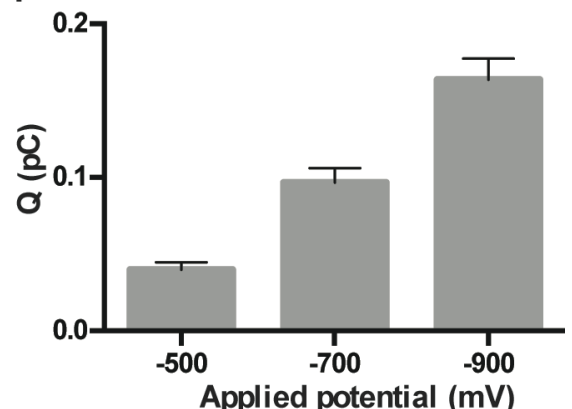

Figure S1. Analysis of the effect of the applied potential on the current spike kinetic and quantitative parameters at amperometric recording of glutamate release from glutamate-filled LUVs. (A) The definitions of the current spike parameters used for analysis. The applied potential effect on (B) spike rise-time (C) spike fall-time (D) spike half-width (E) the maximal current amplitude (F) the total integrated current spike charge. The data are shown as the means of spike kinetics from 5 different glutamate concentrations loaded into LUVs \pm SEM. 


\section{Current spike amplitude and total charge increases with potentials applied.}

The quantitative features $I_{\max }$ and the total integrated charge $(Q)$ of the current spikes detected (Figure S1A) from the recording of LUV glutamate release were then analyzed as the function of applied potential and concentration of encapsulated glutamate solution. The total charge from a single current spike is directly related to the mole of analytes, $N$, that reach the electrode for detection and therefore the number of molecules that reach the electrode surface for detection can be calculated through Faraday's law $(Q=n N F)$ where, $n$ is the number of electrons involved for the reduction reaction ( $n=2$ electrons gained for the reduction reaction of $\mathrm{H}_{2} \mathrm{O}_{2}$ ) and $F$ is Faradays constant (96 $485 \mathrm{C} / \mathrm{mol}$ ). Hence, through the division of $N$ by Avogadro's number $\left(6.022 \times 10^{23} \mathrm{~mol}^{-1}\right)$, the number of $\mathrm{H}_{2} \mathrm{O}_{2}$ molecules detected by the electrode from a single vesicle release event can then be calculated. We observed in these measurements that both $I_{\max }$ and $Q$ increased with a more concentrated encapsulated glutamate concentration in the LUVs and were also significantly affected by the magnitude of the applied potential (Table S2 and Figure S1E,F). This is in agreement with the cyclic voltammograms for the glutamate sensor, ${ }^{11,12}$ which show an increase in current amplitude with change in applied potential from $-0.5 \mathrm{~V}$ to -0.7 and $-0.9 \mathrm{~V}$.

Table S2. Analysis of current spike parameters as a function of the applied potential at the glutamate sensor surface when performing amperometric recording of glutamate release from LUVs pre-filled with various concentrations of glutamate solution.

\begin{tabular}{|c|c|c|c|c|c|c|c|}
\hline $\begin{array}{c}\text { Applied } \\
\text { potential }(\mathrm{mV})\end{array}$ & $\begin{array}{c}\text { Concentration of } \\
\text { glutamate loaded }(\mathrm{mM})\end{array}$ & $\mathrm{T}_{1 / 2}(\mathrm{~ms})$ & $\mathrm{T}_{\text {rise }}(\mathrm{ms})$ & $\mathrm{T}_{\text {fall }}(\mathrm{ms})$ & $I_{\max }(p A)$ & $Q(f C)$ & Spike No. \\
\hline \multirow{5}{*}{-500} & $100(n=4)$ & $0.63 \pm 0.05$ & $0.30 \pm 0.03$ & $0.41 \pm 0.06$ & $34 \pm 5$ & $27 \pm 5$ & 509 \\
\hline & $150(n=4)$ & $0.69 \pm 0.06$ & $0.33 \pm 0.01$ & $0.53 \pm 0.04$ & $35 \pm 3$ & $30 \pm 2$ & 832 \\
\hline & $200(n=4)$ & $0.59 \pm 0.04$ & $0.27 \pm 0.02$ & $0.41 \pm 0.05$ & $54 \pm 4$ & $40 \pm 2$ & 747 \\
\hline & $250(n=5)$ & $0.81 \pm 0.10$ & $0.35 \pm 0.03$ & $0.82 \pm 0.18$ & $44 \pm 8$ & $50 \pm 6$ & 356 \\
\hline & $300(n=4)$ & $0.66 \pm 0.07$ & $0.29 \pm 0.03$ & $0.61 \pm 0.10$ & $47 \pm 4$ & $51 \pm 1$ & 523 \\
\hline \multirow{5}{*}{-700} & $100(n=5)$ & $0.74 \pm 0.04$ & $0.34 \pm 0.03$ & $0.51 \pm 0.03$ & $77 \pm 4$ & $73 \pm 2$ & 628 \\
\hline & $150(n=4)$ & $0.74 \pm 0.09$ & $0.34 \pm 0.06$ & $0.50 \pm 0.10$ & $88 \pm 17$ & $73 \pm 3$ & 948 \\
\hline & $200(n=4)$ & $0.69 \pm 0.04$ & $0.29 \pm 0.02$ & $0.55 \pm 0.04$ & $104 \pm 7$ & $104 \pm 5$ & 460 \\
\hline & $250(n=4)$ & $0.70 \pm 0.06$ & $0.30 \pm 0.03$ & $0.58 \pm 0.07$ & $125 \pm 25$ & $115 \pm 9$ & 448 \\
\hline & $300(n=4)$ & $0.79 \pm 0.09$ & $0.34 \pm 0.01$ & $0.69 \pm 0.11$ & $98 \pm 10$ & $\begin{array}{c}117 \pm 1 \\
2\end{array}$ & 369 \\
\hline \multirow{5}{*}{-900} & $100(n=4)$ & $0.65 \pm 0.06$ & $0.31 \pm 0.07$ & $0.52 \pm 0.02$ & $131 \pm 30$ & $\begin{array}{c}127 \pm 2 \\
6\end{array}$ & 466 \\
\hline & $150(n=4)$ & $0.74 \pm 0.04$ & $0.50 \pm 0.14$ & $0.51 \pm 0.03$ & $145 \pm 26$ & $\begin{array}{c}137 \pm 1 \\
6\end{array}$ & 950 \\
\hline & $200(n=4)$ & $0.67 \pm 0.02$ & $0.36 \pm 0.02$ & $0.40 \pm 0.01$ & $201 \pm 14$ & $\begin{array}{c}170 \pm 1 \\
2\end{array}$ & 1260 \\
\hline & $250(n=4)$ & $0.72 \pm 0.08$ & $0.30 \pm 0.04$ & $0.65 \pm 0.09$ & $202 \pm 37$ & $\begin{array}{c}200 \pm 3 \\
0\end{array}$ & 848 \\
\hline & $300(n=4)$ & $0.76 \pm 0.07$ & $0.41 \pm 0.08$ & $0.61 \pm 0.11$ & $165 \pm 24$ & $185 \pm 7$ & 441 \\
\hline
\end{tabular}

The data are presented as the average of means \pm standard error of the mean (SEM). 


\section{Using glutamate-filled LUVs to calibrate quantitative vesicle glutamate measurements}

Biosensors need calibration to translate the electrical signal recorded into analyte concentration. It is important that the calibration is designed with matching experimental conditions as used for the quantitative measurements. Therefore, we introduced a calibration method for glutamate synaptic vesicle analysis based on probing glutamate release from LUVs that were pre-filled with various glutamate concentrations when performing the glutamate measurement at a fixed potential. As we noted how the amplitude of $I_{\max }$ and the value of $Q$ varies with applied potential, the charge density measured of quantal glutamate release was also evaluated as a function of glutamate concentration and applied potential. The charge density was derived from the detected charge $(Q)$ for the recorded single current spikes and related to the LUV volume that was determined via the measured LUV diameter using nanosized tracking analysis system (Figure 2C). We found that each potential provided its own linear calibration curve (Figure 2D, Figure S2A,B) with all being parallel to one another and differing by the calibration plot intercept, with the $-0.9 \mathrm{~V}$ recording providing the largest and $-0.5 \mathrm{~V}$ providing the lowest, which is in agreement with predictions according to recorded current-voltage plots for $\mathrm{H}_{2} \mathrm{O}_{2}$ (Figure S1E,F)
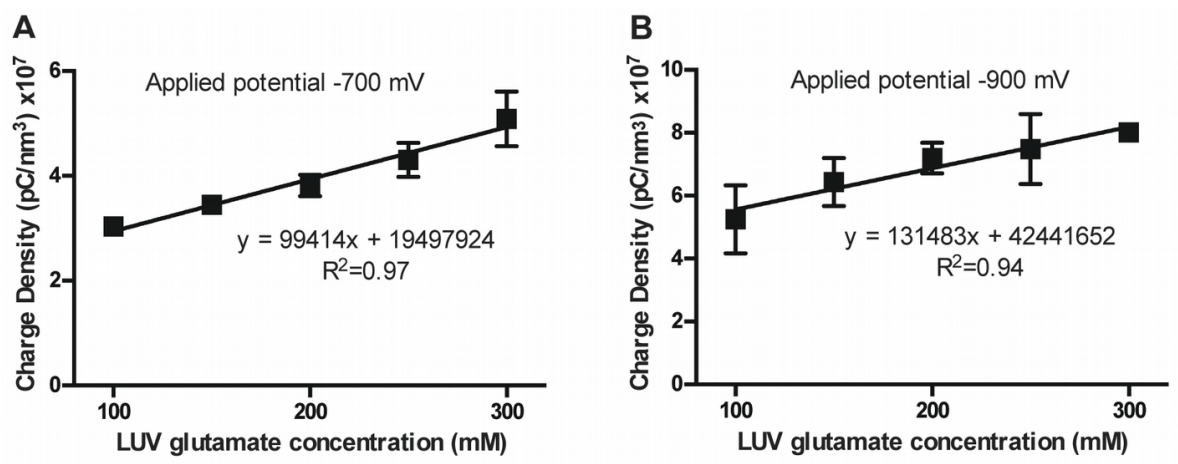

Figure S2. Calibration curve constructed from recording of LUV glutamate release at an applied potential of (A) $-700 \mathrm{mV}$ and $(B)-900 \mathrm{mV}$ vs a $\mathrm{Ag} / \mathrm{AgCl}$ reference electrode. Each data point is an average of charge density from glutamate measurements of LUVs with 5 different glutamate concentration loaded into at one applied potential \pm SEM. 


\section{Using the LUVs calibration for quantification of vesicle glutamate quantal size and exocytotic glutamate release}

After the analysis of the current spikes recorded from glutamate release by glutamate-filled LUVs (Figure 1B), isolated synaptic vesicles (Figure 1C) and exocytotic glutamate release from brain cells in the core region of the nucleus accumbens of mouse (Figure $3 \mathrm{~A}$ ) and rat (Figure S3A) brain tissue, the kinetic and quantitative parameters were analyzed and summarized in Table S5 and S6. Here, the current spikes detected from the exocytosis recordings were divided as categorized by the different dynamic shapes as illustrated in Figure 3A). First by looking at the exocytosis measurements, it shows that the not only the frequency of the different spike types are similar in the two species, but also the temporal value of each category of spikes (i.e. $\mathrm{T}_{1 / 2}, \mathrm{~T}_{\text {rise }}$ and $\mathrm{T}_{\text {fall }}$ ) as well as the quantitative measures $\left(I_{\max }\right.$ and $Q$ ) are very comparable. This implies that the glutamate exocytosis events in mouse and rat brain most probably use very similar release mechanisms that result in similar vesicle quantal size released.

Besides, the current spikes detected when quantifying the glutamate content in isolated synaptic vesicles from mouse brain also present similar current spike dynamics and total current spike charge as recorded from exocytosis events in mouse brain slice. We note that glutamate release from isolated synaptic vesicles bursting, show both a faster $\mathrm{T}_{\text {rise }}$ and slower $\mathrm{T}_{\text {fall }}$ compared to what is detected in exocytosis glutamate release. The slower release kinetics at exocytosis is most likely due to the fact that the dynamics of glutamate release is controlled by the complex SNARE-mediated exocytosis machinery the regulate the formation, dilation and closure of the fusion pore that connects the vesicle compartment to the cell plasma membrane, which is an important feature in regulation of neurotransmitter release and perhaps is the factor behind the resulting different type of categories of current spikes that are detected in these recordings.

The calibration curve obtained from glutamate analysis of the glutamate-filled LUVs were then also used to determine the absolute glutamate quantities of glutamate molecules released from individual exocytosis events during spontaneous glutamate activity in the nucleus accumbens of mouse and brain tissue (Figure 3B, S3B). We noted that the amount glutamate in isolated vesicles was larger than from exocytosis release when events were detected as "sharp spikes" and that the amount was not significantly different for the other complex spike shapes recorded. This difference in quantity between the glutamate stored in isolated synaptic vesicles and the amount released, assuming minimal loss of the vesicle glutamate content at the isolation process, ${ }^{13}$ might stem from different mechanisms of exocytosis present or that glutamate release of more than one vesicle quantal load is detected. ${ }^{11,14}$ 

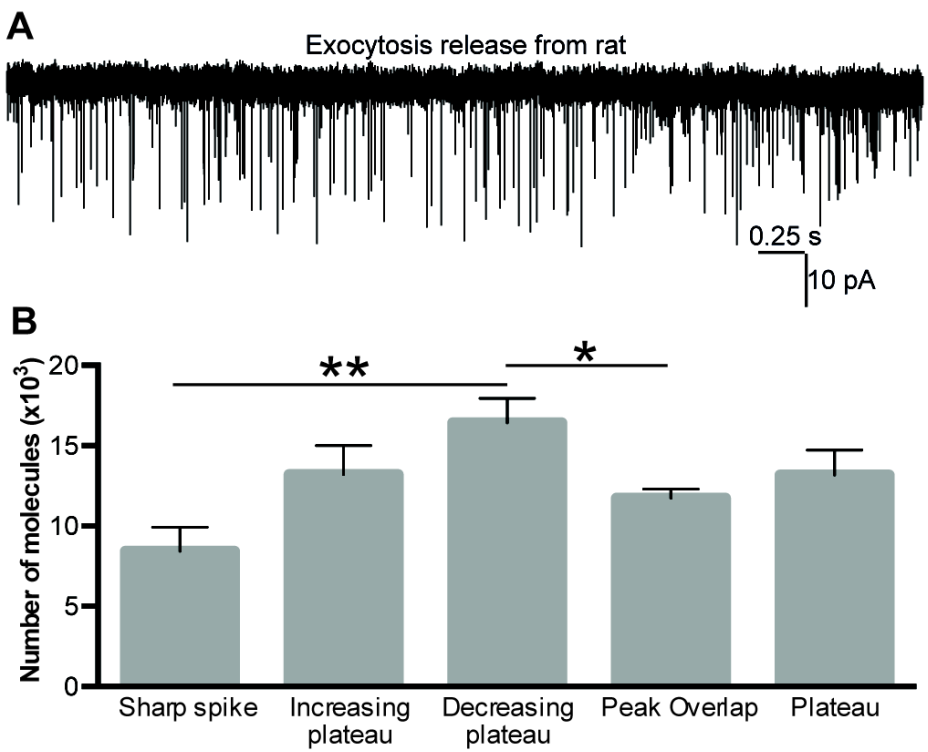

Figure S3. (A) Amperometric recording (10 kHz) of single exocytotic glutamate release in the core region of the nucleus accumbens of rat brain slice using the ultrafast glutamate sensor and applying a $-0.5 \mathrm{~V}$ potential vs a $\mathrm{Ag} / \mathrm{AgCl}$ reference electrode. (B) The number of glutamate molecules released during single exocytosis events was categorized for the different shape of single current spikes recorded. The data is presented as the average number \pm SEM and with a two-tailed paired student's t-test, $* p<0.05, * * p<0.01$.

Table S5 Current spike analysis from amperometric recording of glutamate content in isolated mouse synaptic vesicles and glutamate release from single exocytosis events at the core region of the nucleus accumbens in mouse brain tissue.

\begin{tabular}{|c|c|c|c|c|c|c|c|}
\hline & & $\mathrm{T}_{1 / 2}(\mathrm{~ms})$ & $\mathrm{T}_{\text {rise }}(\mathrm{ms})$ & $\mathrm{T}_{\text {fall }}(\mathrm{ms})$ & $I_{\max }(p A)$ & $\mathrm{Q}(\mathrm{fC})$ & Spike No. \\
\hline \multirow{4}{*}{$\begin{array}{c}\text { Mouse } \\
\text { brain } \\
(n=4)\end{array}$} & Sharp spike & $0.18 \pm 0.07$ & $0.11 \pm 0.03$ & $0.06 \pm 0.01$ & $9.4 \pm 1.6$ & $1.8 \pm 0.3$ & 427 \\
\hline & Increasing plateau & $0.19 \pm 0.07$ & $0.19 \pm 0.08$ & $0.06 \pm 0.02$ & $9.1 \pm 0.77$ & $4.8 \pm 2.7$ & 32 \\
\hline & Peak overlap & $0.24 \pm 0.11$ & $0.13 \pm 0.05$ & $0.15 \pm 0.08$ & $11.5 \pm 2.4$ & $3.2 \pm 0.9$ & 415 \\
\hline & Plateau & $0.26 \pm 0.12$ & $0.12 \pm 0.07$ & $0.22 \pm 0.09$ & $11.1 \pm 1.9$ & $2.9 \pm 1.0$ & 50 \\
\hline \multicolumn{2}{|c|}{$\begin{array}{l}\text { Synaptic vesicle (mouse, } \\
\qquad \mathrm{n}=4)\end{array}$} & $0.16 \pm 0.01$ & $0.08 \pm 0.01$ & $0.14 \pm 0.01$ & $10.87 \pm 0.83$ & $2.2 \pm 0.1$ & 762 \\
\hline \multicolumn{2}{|r|}{ LUVs $(n=21)$} & $0.68 \pm 0.04$ & $0.31 \pm 0.01$ & $0.56 \pm 0.08$ & $42.6 \pm 3.7$ & $39.8 \pm 4.9$ & 2967 \\
\hline
\end{tabular}

The exocytosis data is presented as the average of means \pm SEM. The data from LUVs analysis is the average of averaged values for detection glutamate release from LUVs with 5 different loaded glutamate concentration. 
Table S6. Current spike analysis from amperometric recording of glutamate release from single exocytosis events at the core region of the nucleus accumbens in rat brain tissue

\begin{tabular}{|c|c|c|c|c|c|c|c|}
\hline & & $\mathrm{T}_{1 / 2}(\mathrm{~ms})$ & $\mathrm{T}_{\text {rise }}(\mathrm{ms})$ & $\mathrm{T}_{\text {fall }}(\mathrm{ms})$ & $I_{\max }(p A)$ & $Q(f C)$ & Spike No. \\
\hline \multirow{4}{*}{$\begin{array}{l}\text { Rat } \\
\text { brain } \\
(n=4)\end{array}$} & Sharp spike & $0.46 \pm 0.17$ & $0.19 \pm 0.07$ & $0.13 \pm 0.12$ & $8.6 \pm 2.9$ & $2.7 \pm 0.4$ & 317 \\
\hline & Increasing plateau & $0.80 \pm 0.40$ & $0.79 \pm 0.37$ & $0.13 \pm 0.16$ & $6.5 \pm 2.4$ & $4.2 \pm 0.7$ & 32 \\
\hline & Peak overlap & $0.64 \pm 0.27$ & $0.32 \pm 0.15$ & $0.14 \pm 0.20$ & $8.3 \pm 2.8$ & $3.8 \pm 0.2$ & 332 \\
\hline & Plateau & $0.79 \pm 0.41$ & $0.36 \pm 0.17$ & $0.19 \pm 0.15$ & $10.3 \pm 4.2$ & $4.2 \pm 0.5$ & 101 \\
\hline
\end{tabular}

The data are shown as the means of means of spike kinetics \pm SEM.

\section{References}

(1) Lovrić, J.; Najafinobar, N.; Dunevall, J.; Majdi, S.; Svir, I.; Oleinick, A.; Amatore, C.; Ewing, A. G. On the Mechanism of Electrochemical Vesicle Cytometry: Chromaffin Cell Vesicles and Liposomes. Faraday Discuss. 2016, 193, 65-79.

(2) Riske, K. A.; Dimova, R. Electro-Deformation and Poration of Giant Vesicles Viewed with High Temporal Resolution. Biophys. J. 2005, 88 (2), 1143-1155.

(3) Tsong, T. Y. Electroporation of Cell Membranes. Biophys. J. 1991, 60 (2), 297-306.

(4) Dunevall, J.; Fathali, H.; Najafinobar, N.; Lovric, J.; Wigström, J.; Cans, A.-S.; Ewing, A. G. Characterizing the Catecholamine Content of Single Mammalian Vesicles by Collision-Adsorption Events at an Electrode. J. Am. Chem. Soc. 2015, 137 (13), 4344-4346.

(5) Wang, C.; Ye, F.; Velardez, G. F.; Peters, G. H.; Westh, P. Affinity of Four Polar Neurotransmitters for Lipid Bilayer Membranes. J. Phys. Chem. B 2011, 115 (1), 196-203.

(6) Lipowsky, R. Bending of Membranes by Anchored Polymers. Europhys. Lett. 1995, 30 (4), 197-202.

(7) Simon, J.; Kühner, M.; Ringsdorf, H.; Sackmanna, E. Polymer-Induced Shape Changes and Capping in Giant Liposomes. Chem. Phys. Lipids 1995, 76 (2), 241-258.

(8) Döbereiner, H.-G.; Selchow, O.; Lipowsky, R. Spontaneous Curvature of Fluid Vesicles Induced by Trans-Bilayer Sugar Asymmetry. Eur. Biophys. J. 1999, 28 (2), 174-178.

(9) Kakorin, S.; Neumann, E. Kinetics of the Electroporative Deformation of Lipid Vesicles and Biological Cells in an Electric Field. Berichte der Bunsengesellschaft für Phys. Chemie 1998, 102 (4), 670-675.

(10) Reimhult, E.; Höök, F.; Kasemo, B. Intact Vesicle Adsorption and Supported Biomembrane Formation from Vesicles in Solution: Influence of Surface Chemistry, Vesicle Size, Temperature, and Osmotic Pressure †. Langmuir 2003, 19 (5), 1681-1691.

(11) Wang, Y.; Mishra, D.; Bergman, J.; Keighron, J. D.; Skibicka, K. P.; Cans, A.-S. Ultrafast Glutamate Biosensor Recordings in Brain Slices Reveal Complex Single Exocytosis Transients. ACS Chem. Neurosci. 2019, 10 (3), $1744-1752$.

(12) Keighron, J. D.; Wigström, J.; Kurczy, M. E.; Bergman, J.; Wang, Y.; Cans, A.-S. Amperometric Detection of Single Vesicle Acetylcholine Release Events from an Artificial Cell. ACS Chem. Neurosci. 2015, 6 (1), 181-188.

(13) Carlson, M. D.; Ueda, T. Accumulated Glutamate Levels in the Synaptic Vesicle Are Not Maintained in the Absence of 
Active Transport. Neurosci. Lett. 1990, 110 (3), 325-330.

(14) Helassa, N.; Dürst, C. D.; Coates, C.; Kerruth, S.; Arif, U.; Schulze, C.; Wiegert, J. S.; Geeves, M.; Oertner, T. G.; Török, K. Ultrafast Glutamate Sensors Resolve High-Frequency Release at Schaffer Collateral Synapses. Proc. Natl. Acad. Sci. 2018, 115 (21), 5594-5599.

(15) Mayer, L. D.; Hope, M. J.; Cullis, P. R.; Janoff, A. S. Solute Distributions and Trapping Efficiencies Observed in FreezeThawed Multilamellar Vesicles. Biochim. Biophys. Acta - Biomembr. 1985, 817 (1), 193-196.

(16) Finot, M. O.; Braybrook, G. D.; Mcdermott, M. T. Characterization of Electrochemically Deposited Gold Nanocrystals on Glassy Carbon Electrodes. J. Electroanal. Chem. 1999, 466 (2), 234-241.

(17) Ahmed, S.; Holt, M.; Riedel, D.; Jahn, R. Small-Scale Isolation of Synaptic Vesicles from Mammalian Brain. Nat. Protoc. 2013, 8 (5), 998-1009.

(18) Budzinski, K. L.; Allen, R. W.; Fujimoto, B. S.; Kensel-Hammes, P.; Belnap, D. M.; Bajjalieh, S. M.; Chiu, D. T. Large Structural Change in Isolated Synaptic Vesicles upon Loading with Neurotransmitter. Biophys. J. 2009, 97 (9), 25772584.

(19) Mosharov, E. V; Sulzer, D. Analysis of Exocytotic Events Recorded by Amperometry. Nat. Methods 2005, 2, 651. 
